\title{
Structure and Mechanical Properties of Sequential Interpenetrating Polymer Networks. I. Poly(ethyl acrylate)/Poly(methyl methacrylate) System
}

\author{
Hiroshi ADACHI* and Tadao KoTAKA** \\ Department of Macromolecular Science, Faculty of Science, \\ Osaka University, Toyonaka, Osaka 560, Japan.
}

(Received December 25, 1981)

\begin{abstract}
Interpenetrating polymer networks (IPNs) composed of poly(ethyl acrylate) (PEA) and poly(methyl methacrylate) (PMMA) were prepared by two different methods of composition control: control of the swelling ratio of the first PEA network with a MMA second monomer mixture subsequently allowed to polymerize and become crosslinked upto the point of completion (SW-series), and control of the conversion of MMA absorbed in the PEA network (CVseries). The dynamic mechanical properties and stress-strain behavior of these IPNs were examined. In the process of IPN formation, it is likely that the MMA first forms microgels in the PEA network, and these microgels eventually interconnect to form a fully interpenetrating network having microheterogeneous nodular domains. The glassy PMMA microdomains impart a filler effect (similar to that found in filled elastomers). Semi- and full-IPNs of $75 \mathrm{wt} \%$ PMMA content in which PMMA was uncrosslinked and crosslinked, respectively, were also prepared and the mechanical properties were examined. Viscoelastic behavior of both semi- and full-IPNs is linear with small deformation, but nonlinear when subjected to large and/or high speed deformation. The full-IPN was stronger and stiffer than the semi-IPN.
\end{abstract}

KEY WORDS Poly(ethyl acrylate) / Poly(methyl methacrylate) / Interpenetrating Polymer Network / Nodular Structure / Filler Effect / Thermorheologically Simple / Nonlinear Creep / Stress-Strain Behavior / Failure Envelope /

Since 1960 when interpenetrating polymer networks (IPNs) were first prepared by Millar, ${ }^{1}$ a number of studies have been reported on various IPNs. $^{2-13}$ Interest in regard to these studies has been directed primarily toward the fact that an IPN is a kind of polymer blend for obtaining a compatible material from polymers mutually immiscible. In the literature, several different methods for preparing IPNs have been reported. One of these is to prepare what are usually called sequential interpenetrating polymer networks (SIPNs). For preparing these SIPNs, a crosslinked polymer (I) is swollen with a second monomer (II) containing a crosslinking agent and an initiator, and the second monomer mixture is allowed to polymerize and crosslink in situ. Among SIPNs, an IPN consisting of fully crosslinked first and second components is designated as a full-IPN, while an IPN consisting of one crosslinked and one uncrosslinked component each is designated as either a semi-IPN or a quasi-IPN. ${ }^{4}$

The structural features of SIPNs reported so far are summarized as follows. ${ }^{3-6}$ (i) The two polymer components are fairly well mixed with each other, but are usually phase-separated to some extent. ${ }^{10-13}$ (ii) The two components usually form two mutually continuous phases, but the phase of the first network is predominant. ${ }^{10-13}$ (iii) The

* Present Address: Manufacturing Development Laboratory, Mitsubishi Electric Corporation, Amagasaki, Hyogo 661, Japan.

** To whom correspondence should be addressed. 
domain size is controlled primarily by the crosslinking density of the first network. The greater the degree of crosslinking, the finer is the domain size of the second network. ${ }^{12,13}$ Therefore, it is quite likely that the structure and properties of an SIPN depend largely on the synthetic method rather than on the mutual immiscibility of the parent polymers. We have carried out a study to elucidate the synthesismorphology-property relationships in greater detail, using SIPNs consisting of a lesser compatible pair, a semicompatible pair, and a highly compatible, complex-forming pair as the constituent polymers. This article is concerned with an SIPN composed of a semicompatible pair of poly(ethyl acrylate) and poly(methyl methacrylate) as the first (I) and the second (II) components, respectively.

Some data are already available on the blends and SIPNs of the PEA/PMMA system. The heat of mixing of PEA and PMMA was reported to be nearly zero. ${ }^{14}$ A blend of PEA/PMMA (79:21 by weight) was reported to exhibit a single very broad glass-to-rubber transition region; this behavior was attributed to partial miscibility of the two components. ${ }^{15}$ However, when such a blended specimen was annealed at a temperature above the glass transition temperature, $T_{\mathrm{g}}$, of PMMA, partial phase separation occurred and the specimen became opaque. $^{16}$ These results indicate that the PEA/ PMMA system is partly miscible or semicompatible, and may undergo a lower-critical-solutiontemperature (LCST) type phase separation. Sperling et $a .^{7-11}$ prepared SIPNs of PEA/ PMMA by a photopolymerization $\operatorname{method}^{7-10}$ and observed a very broad continuous transition and significant phase separation still present. ${ }^{10-11}$

The purpose of this study has been primarily (i) to find how a mutually continuous two phase structure develops, (ii) to examine the two phase structure in terms of dynamic viscoelastic properties, and finally (iii) to examine how the morphology, especially that of the second PMMA network effects the tensile properties of the SIPNs. Thus, we prepared full-SIPNs of PEA (I) and PMMA (II) by two different methods. In one method, the ratio of PEA/ PMMA was controlled by adjusting the swelling ratio of the (I) network by the (II) monomer subsequently allowed to polymerize and crosslink to completion. In the other method, the ratio of PEA/ PMMA was controlled by adjusting the monomerto-polymer conversion of the (II) monomer fully absorbed in advance by the (I) network. The former SIPNs were designated as the SW-series and the latter the CV-series. For comparison, we prepared a semi-IPN from a fully swollen PEA network with a MMA monomer containing only an initiator and allowed to completely polymerize. Conclusions drawn from such a study may apply to other SIPNs composed of semicompatible polymer pairs.

\section{EXPERIMENTAL}

\section{Materials}

An uncrosslinked PEA prepolymer was prepared by the solution polymerization method. ${ }^{17}$ The molecular weight $M_{w}$ and $M_{w} / M_{n}$ were $46 \times 10^{4}$ and 2.4 , respectively, as determined by gel permeation chromatography (GPC; Toyo Soda Ltd. Model HLC-801A) with a built-in refractometer. The carrier was chloroform. Commercially available polystyrenes (Toyo Soda Ltd., TSK PSs) were used as elution standards. GPC was carried out with a flow-rate of $1.0 \mathrm{ml} \mathrm{min}-1$ at $40^{\circ} \mathrm{C}$. The polymer concentration was $0.03 \%(\mathrm{w} / \mathrm{v})$.

The PEA prepolymer was crosslinked by the following procedure. The polymer was mixed with $2 \mathrm{wt} \%$ benzoyl peroxide (BPO) in benzene. The benzene solution was cast on glass to obtain films of $0.3 \mathrm{~mm}$ thick. The films were cured at $120^{\circ} \mathrm{C}$ under a pressure of $3.5 \mathrm{MPa}$ for $90 \mathrm{~min}$ in a laboratory hot press. Remaining uncrosslinked PEA fractions were extracted by refluxing with benzene for $6 \mathrm{~h}$. The crosslinked PEA films had an effective network chain density of $26 \mathrm{~mol} \mathrm{~m}^{-3}$, when evaluated by Young's modulus $E$ at $18^{\circ} \mathrm{C}$ which was $0.19 \mathrm{MPa}$. These films were used as the (I) network for subsequent preparation of IPNs.

For the IPN preparation, a MMA monomer was mixed with $0.1 \mathrm{~mol} \%$ ethylene dimethacrylate (EDMA) as the crosslinking agent and $1 \mathrm{wt} \% 2,2^{\prime}$ azobisisobutylonitrile (AIBN) as the initiator. The final PEA/PMMA content was adjusted by the two methods described in INTRODUCTION. Thus, for SW-series IPNs, crosslinked PEA films were immersed in the MMA mixture for various periods of time to adjust the swelling ratio. It took only $5 \mathrm{~min}$ at $16^{\circ} \mathrm{C}$ for the PEA network to reach a swelling-equilibrium of about $75 \mathrm{wt} \%$ MMA content or a maximum swelling ratio of 3 . Each swollen film was placed in an air-tight polymerization cell. The film was allowed to stand at room 
a) SW-IPN

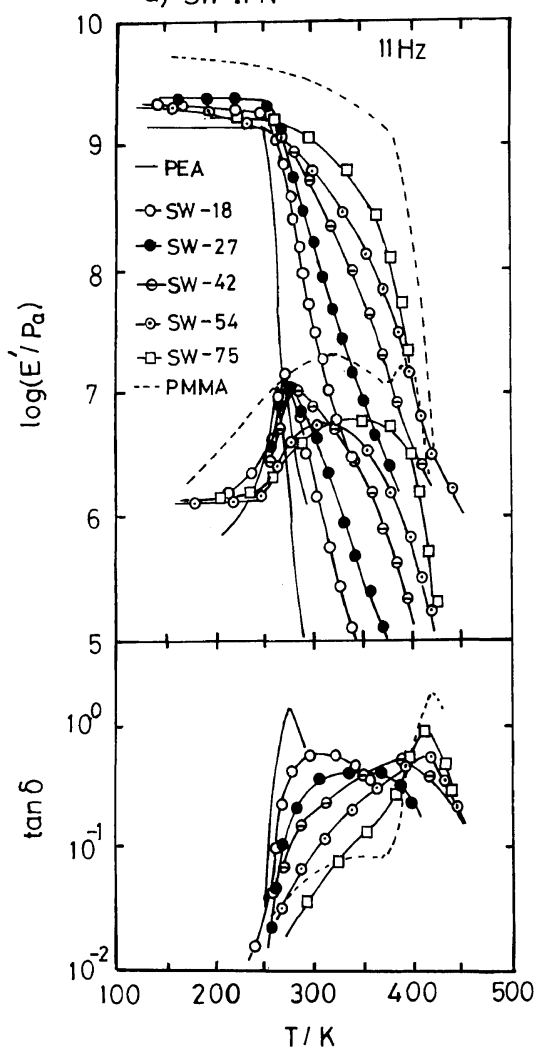

b) CV-IPN

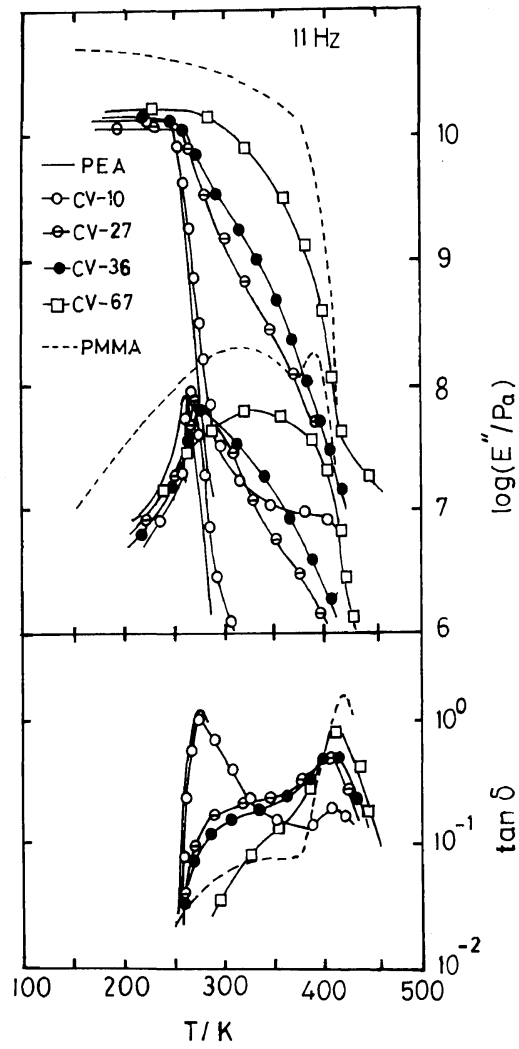

Figure 1. Storage $\left(E^{\prime}\right)$, loss $\left(E^{\prime \prime}\right)$ moduli and loss tangents $(\tan \delta$ ) plotted against temperature for (a) SW- and (b) CV-series full-IPNs, determined at $11 \mathrm{~Hz}$ with heating rate of $1 \mathrm{~K} \mathrm{~min}{ }^{-1}$. Curves for homopolymer networks are also shown for comparison.

reached a maximum after about $30 \mathrm{~min}$ or at $15 \%$ conversion and became nearly zero after about $100 \mathrm{~min}$ or at $90 \%$ conversion.

Figure 1 compares the temperature dependence of tensile storage moduli $E^{\prime}$, loss moduli $E^{\prime \prime}$, and loss tangents $\tan \delta$ for the SW- and CV-series full IPNs. Figure 1a shows that all SW-IPNs exhibit only one peak in the $E^{\prime \prime}$ and $\tan \delta$ curves, and a one-step decrease in the $E^{\prime}$ curve. With increasing PMMA content, the loss maximum temperature $T_{\max }$ shifts to higher temperature, and the peak becomes broader. Finally, the peak becomes very broad covering a temperature range between the $T_{\mathrm{g}}$ 's of the constituent polymers. Correspondingly, the decrease in $E^{\prime}$ becomes gradual over the same range of temperature. The $\tan \delta$ peak also gradually shifts to higher temperature, and a tail appears on the lower temperature side of the peak for PMMA content higher than $40 \mathrm{wt} \%$.

On the other hand, Figure 1b shows that the CVIPNs appear to have a wider transition than do the corresponding SW-IPNs. The $E^{\prime \prime}$ and $\tan \delta$ curves of CV-10 exhibit double peaks at about the $T_{\max }$ of PEA and PMMA. The $E^{\prime}$ curve of the same sample exhibits a two-step decrease with increasing temperature. The $E^{\prime}$ and $E^{\prime \prime}$ curves of other CV-IPNs were substantially similar to those of the corresponding SW-IPNs. However, as for $\tan \delta$, there was a significant difference. The $\tan \delta$ curves of CVIPNs have two peaks or one peak and an accompanying shoulder at low temperature. With increasing PMMA content, the height of the high $T_{\max }$ peak increases, while the low $T_{\max }$ peak shifts to high temperature eventually diminishing to a shoulder. Sperling et al. ${ }^{7-10}$ also reported that their PEA/ PMMA IPNs exhibited very broad transition re- 


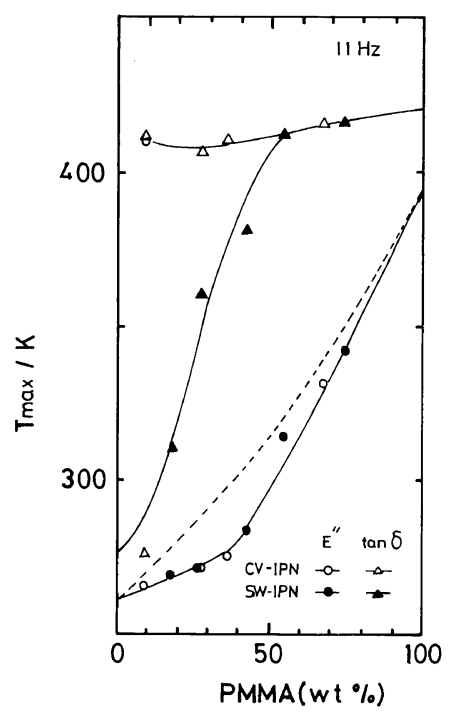

Figure 2. Comparison between the loss $E^{\prime \prime}$ and $\tan \delta$ maximum temperatures $\left(T_{\max }\right.$ 's) $v s$. composition in weight $\%$ of PMMA for SW- and CV-series full IPNs. Broken curve represents the Fox equation of $T_{\mathrm{g}}{ }^{18}$

gions. However, the detailed features of their results are somewhat different from ours. The broad transition appears to be characteristic of IPNs composed of high and low $T_{\mathrm{g}}$ components.

Figure 2 summarizes the composition dependence of the loss and $\tan \delta$ maximum temperatures $T_{\max }$ for SW- and CV-IPNs. The loss maximum temperatures of these IPNs exhibit a nearly identical dependence on PMMA content, except for CV-10 which displays two peaks. However, there can be seen a significant difference in the $\tan \delta$ maximum temperatures. The three CV-IPNs with low PMMA content have two distinct $T_{\max }$. The broken curve represents the Fox equation ${ }^{18}$ of $T_{\mathrm{g}}$ proposed for random copolymers and compatible blends. Its discrepancy with our data is greater at lower PMMA content.

In Figure 3, the $E^{\prime}$ values for SW- and CV-IPNs determined at $303 \mathrm{~K}$ are compared with two mixture rules. One is a three dimensional Takayanagi model proposed by Kraus and Rollman, ${ }^{19}$ and the other is the $1 / 5$ power law model proposed by Davies. ${ }^{20}$ These models may be applied to mutuallycontinuous two-phase systems such as the present IPNs. In Figure 3, the shaded zone represents the Kraus model with the lower bound:

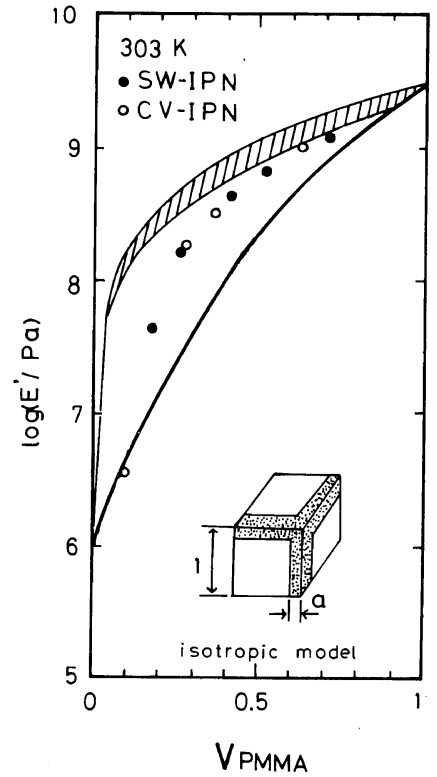

Figure 3. Young's moduli $\left(E^{\prime}\right)$ observed at $303 \mathrm{~K} v$ s. composition in volume fraction of PMMA $\left(V_{\mathrm{PMMA}}\right)$ for SW- and CV-series full-IPNs. Shaded area indicates the values calculated from the isotropic model, eq 1 and 2, and solid curve Davies' 1/5 power model, eq 3 .

$$
E=(1-a)^{2} E_{1}+a^{2} E_{2}+2 a(1-a)\left[\frac{1-a}{E_{1}}+\frac{a}{E_{\mathrm{a}}}\right]^{-1}
$$

and the upper bound:

$$
E=\left[\frac{a}{a(2-a) E_{2}+(1-a)^{2} E_{1}}+\frac{1-a}{a^{2} E_{2}+\left(1-a^{2}\right) E_{1}}\right]^{-1}
$$

The volume fraction $V_{2}$ of component 2 is related to the fraction $a$ as $V_{2}=a^{2}(3-2 a)$. The solid curve is the Davies model:

$$
E^{1 / 5}=V_{1} E_{1}{ }^{1 / 5}+V_{2} E_{2}{ }^{1 / 5}
$$

In all these equations, $E$ and $E_{i}(i=1$ or 2) are Young's moduli of the composite and the component $i$, respectively.

The Davies equation was originally derived for predicting the elastic modulus of semicrystalline polymers, which may be best modelled by amorphous and crystalline two-phase mixtures with two continuous phases. ${ }^{20-23}$ It was found to hold very well for moduli differing by a factor of as much as one thousand..$^{23}$ The Davies model has been found to hold also for interstitial polymers such as PMMA-modified polyurethane elastomers, ob- 


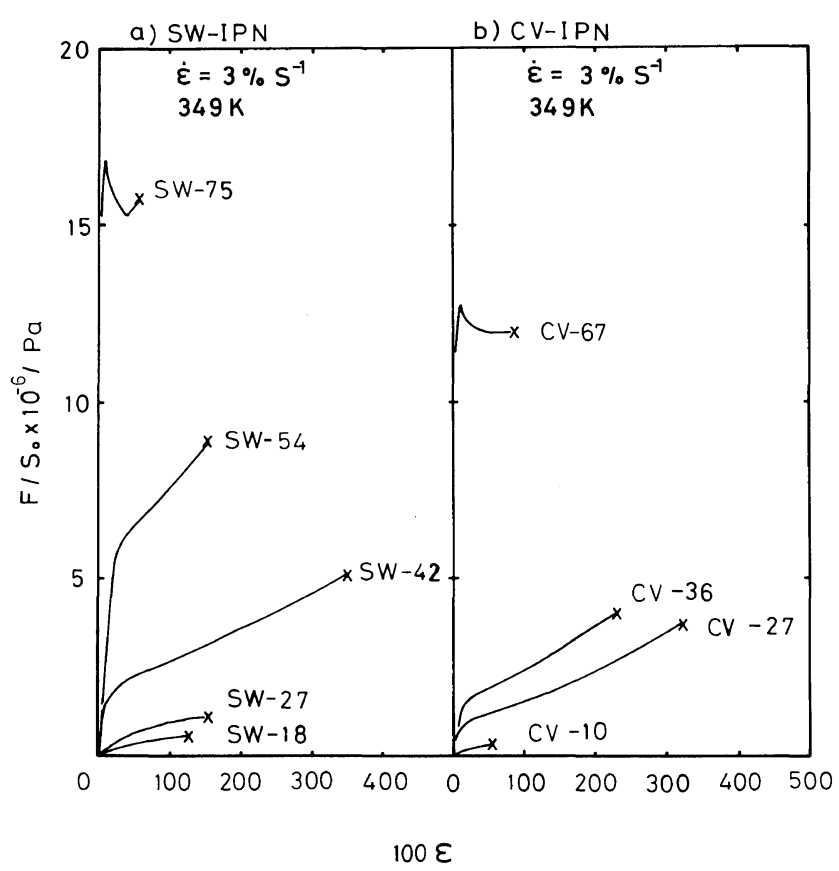

Figure 4. Stress-strain curves for (a) SW-and (b) CV-series full-IPNs obtained at $349 \mathrm{~K}$ with strain rate of $3 \% \mathrm{~s}^{-1}$. Marks $(\times)$ denote break points.

tained by polymerizing MMA in a preformed polyurethane network. ${ }^{21}$ Therefore, the interstitial polymers are a kind of semi-IPNs. In contrast with conventional rubber-modified plastics such as high-impact polystyrenes, these polymers have two continuous phases. Davies et al. ${ }^{21,22} \mathrm{em}-$ phasized that their model accounts for the particle-particle or phase-phase interactions through the interfacial layer. The model was particularly successful in predicting polyurethane based semi-IPNs. ${ }^{21,22}$

The Kraus-Rollman model ${ }^{19}$ is an extension of the Takayanagi model ${ }^{24}$ to a three-dimensional case. Thus, this model might better apply to twophase systems in which only one phase is continuous. ${ }^{23}$ In the Takayanagi model, the phasephase interactions have not been specifically considered. ${ }^{21,22}$ It is interesting to note that the elastic moduli of the present IPNs appear to fall between the values predicted by these two models.

Figure 4 shows stress-strain curves obtained at $349 \mathrm{~K}$ with a constant strain rate of $3 \% \mathrm{~s}^{-1}$. With increasing PMMA content, a change from rubbery to plastic behavior is seen for both SW- and CV-
IPNs with high PMMA content. Finally, a yielding phenomenon is observed for the former of $75 \mathrm{wt} \%$ PMMA, and for the latter of $67 \mathrm{wt} \%$ PMMA.

Figure 5 shows the composition dependence of the tensile strength $\sigma_{\mathrm{b}}$ and the strain-at-break $\varepsilon_{\mathrm{b}}$ at $349 \mathrm{~K}$ with $\dot{\varepsilon}=3 \% \mathrm{~s}^{-1}$ for SW- and CV-IPNs. For SW-IPNs, $\varepsilon_{\mathrm{b}}$ possesses a maximum at about $40 \mathrm{wt} \%$ PMMA. On the other hand, for CV-IPNs, $\varepsilon_{\mathrm{b}}$ possesses a maximum at about $30 \mathrm{wt} \%$ PMMA. The difference in the ultimate properties may be interpreted as due to the difference in microheterogeneity between SW- and CV-IPNs.

These results suggest that the PEA/PMMA IPNs with PMMA content higher than $40 \mathrm{wt} \%$ have two mutually continuous phases and that the two components are fairly well mixed or interpenetrate with each other. The CV-IPNs, especially with low PMMA content, appear to have PMMA microdomains dispersed in a continuous PEA network phase. Such glassy microdomains may act as fillers similar to those in filled elastomers. Thus, these CVIPNs with low PMMA content are superior to the corresponding SW-IPNs with respect to modulus, tensile strength, and strain-at-break. 


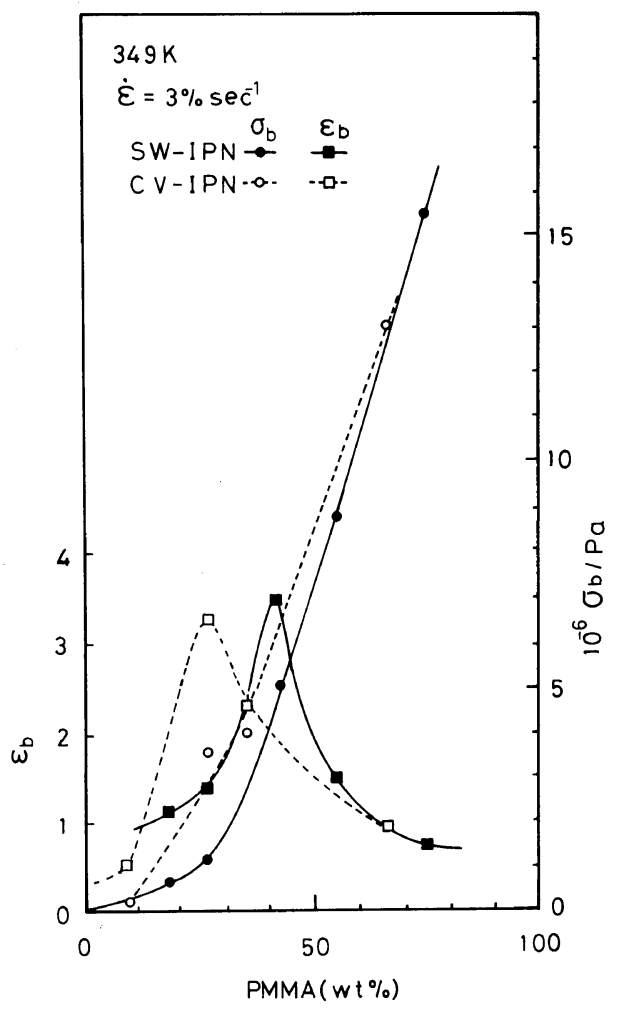

Figure 5. Plots of tensile strength $\sigma_{\mathrm{b}}$ and strain at break $\varepsilon_{\mathrm{b}} v s$. composition in weight $\%$ of PMMA for SWand CV-series full-IPNs determined at $349 \mathrm{~K}$ with strain rate of $3 \% \mathrm{~s}^{-1}$.

\section{Properties of Semi- and Full-IPNs}

Next, our attention was directed to the difference between semi- and full-IPNs with the same $75 \mathrm{wt} \%$ PMMA content coded, respectively, as SW-75s and SW-75. Figure 6 shows the temperature dependence of $E^{\prime}$ and $E^{\prime \prime}$ for SW-75s and SW-75. The two curves are quite similar to each other. Figure 7 shows the tensile stress-relaxation master curves for $\mathrm{SW}-75 \mathrm{~s}$ and SW-75. The reference temperature $T_{\mathrm{r}}$ is $393 \mathrm{~K}$ for both curves. Apparently, the timetemperature superimposition holds well for these curves which show similar broad transitions. Temperature dependence of $\log a_{\mathrm{T}}$ for SW-75s and SW-75 is shown in the inserts of the figures. The solid curves represent the WLF equation ${ }^{25}$ with $T_{\mathrm{r}}=393 \mathrm{~K}$. It is seen that $\log a_{\mathrm{T}}$ for both SW-75s and SW-75 falls on the WLF curve except at low temperatures.

Figure 8 shows the creep compliances $J(\sigma, t)$ at

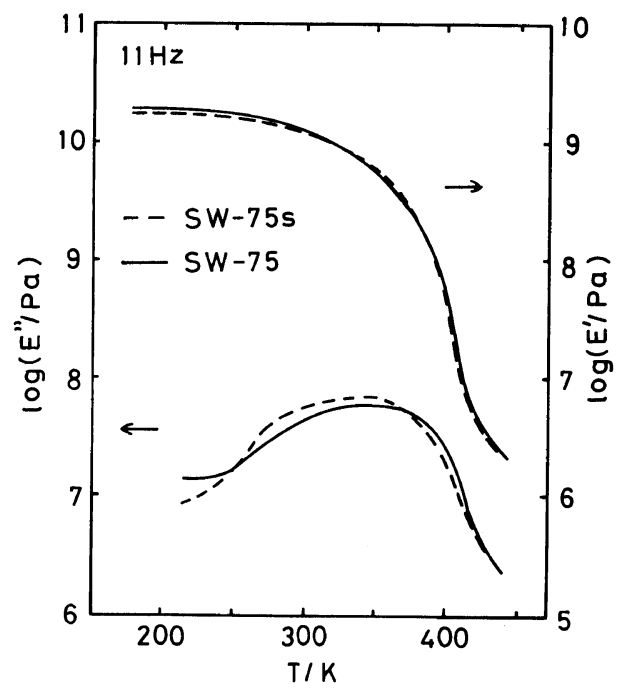

Figure 6. Storage $\left(E^{\prime}\right)$, loss $\left(E^{\prime \prime}\right)$ moduli vs. temperature for semi- and full-IPNs, SW-75s and SW-75, respectively, determined at $11 \mathrm{~Hz}$ with heating rate of $1 \mathrm{~K}$ $\min ^{-1}$.

$368 \mathrm{~K}$ for SW-75s and SW-75. We note that $J$ depends on the stress $\sigma$. As seen in Figure 8a, $J(\sigma, t)$ for SW-75s deviates from linearity even under stress as small as $0.93 \mathrm{MPa}$ (or $\log J(\sigma, t)$ below $10^{-8}$ $\left.\mathrm{Pa}^{-1}\right)$. For $\sigma$ greater than $3 \mathrm{MPa}$, the curve becomes slightly sigmoidal. On the other hand, three $J(\sigma, t)$ curves for SW-75 for $\sigma$ below 1.3 MPa exhibit no deviation from linearity. With increasing $\sigma$, the $J(\sigma, t)$ curves become distinctly sigmoidal, and more rapidly approach the asymptote $\left(J_{\infty}=4.7 \times 10^{-7}\right.$ $\left.\mathrm{Pa}^{-1}\right)$.

$J_{\infty}$ is roughly the same as that of SW-75s. However, at $413 \mathrm{~K}$, which is well above the $T_{\mathrm{g}}$ of PMMA, both SW-75s and SW-75 exhibit nearly the same linear creep behavior as crosslinked rubber.

Figures 9, 10, and 11 shows stress-strain curves for semi- and full-IPNs of different temperatures at a fixed strain rate of $3 \% \mathrm{~s}^{-1}$, and those at varying strain rates at $368 \mathrm{~K}$ and $413 \mathrm{~K}$, respectively. In obtaining these data, note of the specimen showed necking but were extended uniformly at all times. With increasing temperature, either specimen showed a plastic-to-rubber transition. At temperatures below $373 \mathrm{~K}$, stress-whitening was observed for both specimens.

Figure 12 shows plots of $\sigma_{\mathrm{b}}$ and yield strength $\sigma_{\mathrm{y}}$ against the logarithm of strain rate $\dot{\varepsilon}$ for SW-75s 


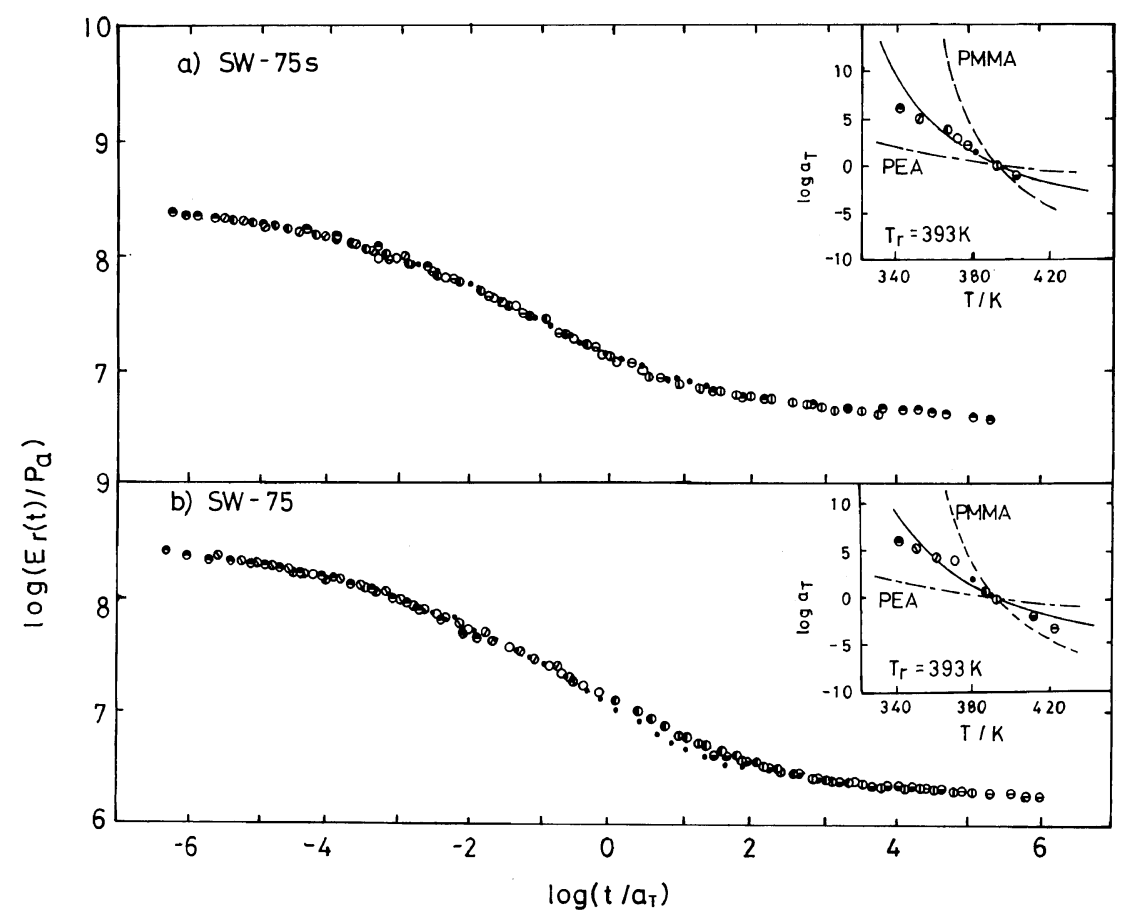

Figure 7. Tensile stress-relaxation master curves for (a) semi-IPN (SW-75s) and (b) full-IPN (SW-75) reduced to $T_{\mathrm{r}}=393 \mathrm{~K}$. Data points include those obtained in the range of strain $0.015-0.04$ over a temperature range from 343 to $423 \mathrm{~K}$. The initial strain rate was $390 \% \mathrm{~s}^{-1}$. Curves in the inserts represent the WLF equations for PMMA, PEA, and IPNs.

and SW-75; these were derived from Figure 10. Apparently, SW-75 possesses a larger tensile strength; the breaking strength is dependent on the degree of crosslinking.

On the other hand, the yield stress at low strain rates is almost the same for both SW-75s and SW75. However, at higher strain rates where distinct yield points can be observed, the value of $\sigma_{\mathrm{y}}$ for SW75 becomes larger than that of SW-75s.

These tensile strength data are summarized in Figure 13 in the form of reduced failure envelopes as defined by Smith, ${ }^{26}$ where $\log \left(\sigma_{\mathrm{b}} T_{0} / v T\right)$ is plotted against $\log \left(100 \varepsilon_{\mathrm{b}}\right)$, where $T_{0}=413 \mathrm{~K}$ is an arbitrarily taken reference temperature and $v$ is the network chain density determined form Young's modulus value ( $c f$. Table II). The shaded area in the figure is the region where most of the data for different singly-component elastomers with varying $v$ and $T$ were obtained. ${ }^{27}$ The data for homo-PEA networks and those for SW-75s and SW-75 at low strain rates also fall within this shaded region.
However, the data points for IPNs show that SW-75 is the stiffest and SW-75s is next to this according to high speed tensile tests.

\section{DISCUSSION}

\section{Structures of $S W$ - and CV-PEA/PMMA SIPNS}

The primary relaxation of poly(alkyl methacrylate)s is usually obscured by the accompanying large secondary relaxation of the side groups. Therefore, it is difficult to estimate the extent of the intermixing of the PEA and PMMA phases from the primary relaxation data of the IPNs. However, looking carefully at the viscoelastic properties of the IPNs, we may deduce the following conclusions. Namely, the viscoelastic properties of the CV- and SW-series IPNs suggest that a microheterogeneous structure is formed especially in the early stage of the (II) network formation. In the kinetics of the (II) network formation in the (I) network, an acceleration of the reaction began at 
Structure and Properties of PEA/PMMA SIPN

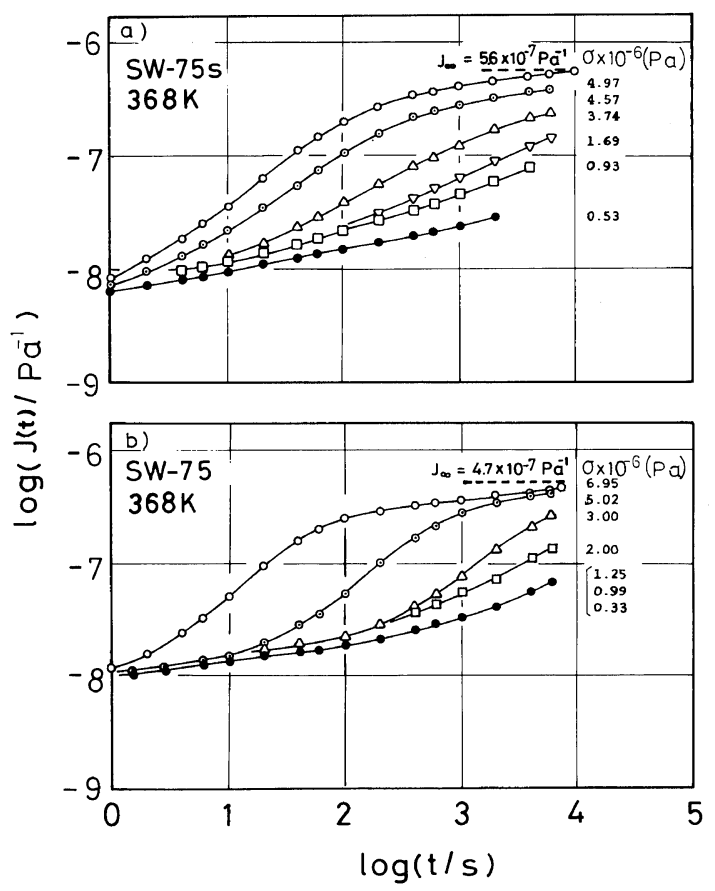

Figure 8. Nonlinear creep compliances $J(\sigma, t)$ for (a) semi-IPN (SW-75s) and (b) full-IPN (SW-75) obtained at $368 \mathrm{~K}$ under the various constant stresses indicated.

a) $5 w-75 \mathrm{~s}$

b) SW -75

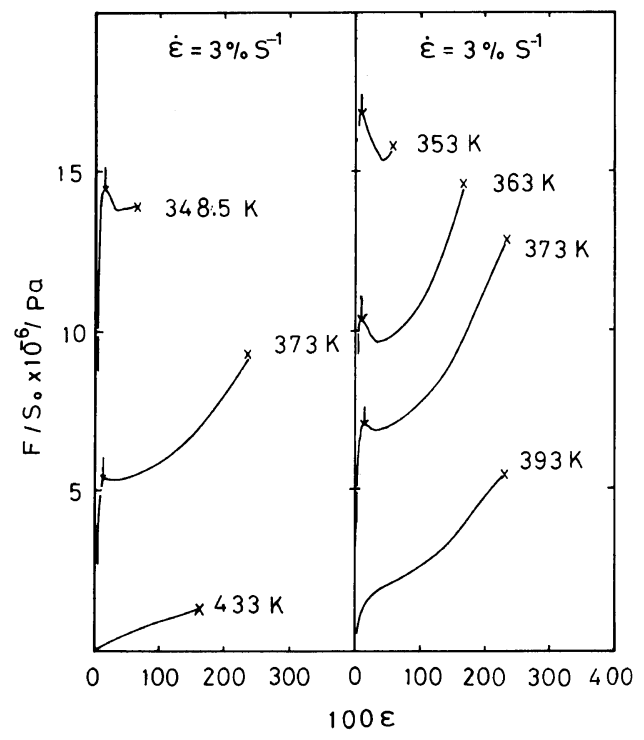

Figure 9. Stress-strain curves for (a) semi-IPN (SW75s) and (b) full-IPN (SW-75) obtained at the various temperatures indicated. Strain rate $\varepsilon$ was $3 \% \mathrm{~s}^{-1}$. Arrows denote yield points and marks $(x)$ break points.

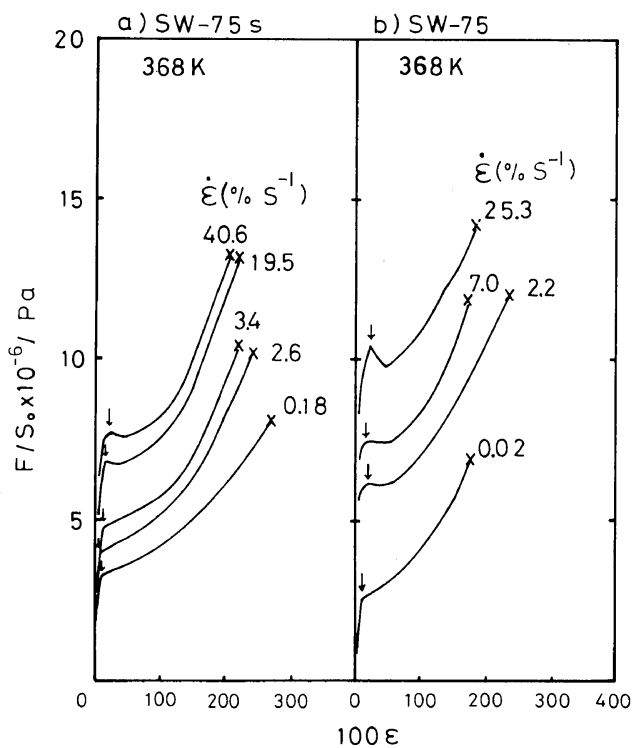

Figure 10. Stress-strain curves for semi-IPN (SW-75s) and full-IPN (SW-75) obtained at $368 \mathrm{~K}$ with various strain rates $\varepsilon$ as indicated. Marks $(\times)$ denote break points and arrows yield points.

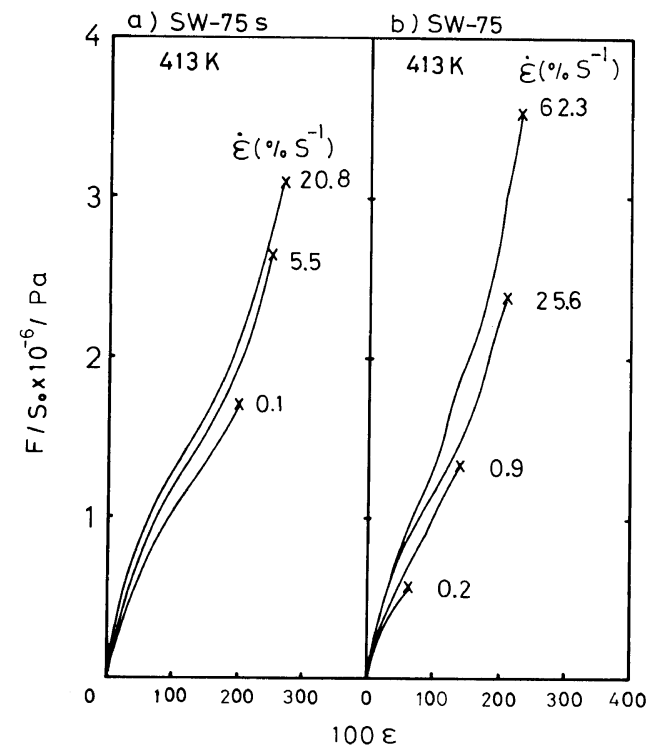

Figure 11. Stress-strain curves for (a) semi-IPN (SW$75 \mathrm{~s}$ ) and full-IPN (SW-75) obtained at $413 \mathrm{~K}$ with various strain rates $\varepsilon$ as indicated. Marks $(x)$ denote break points. 


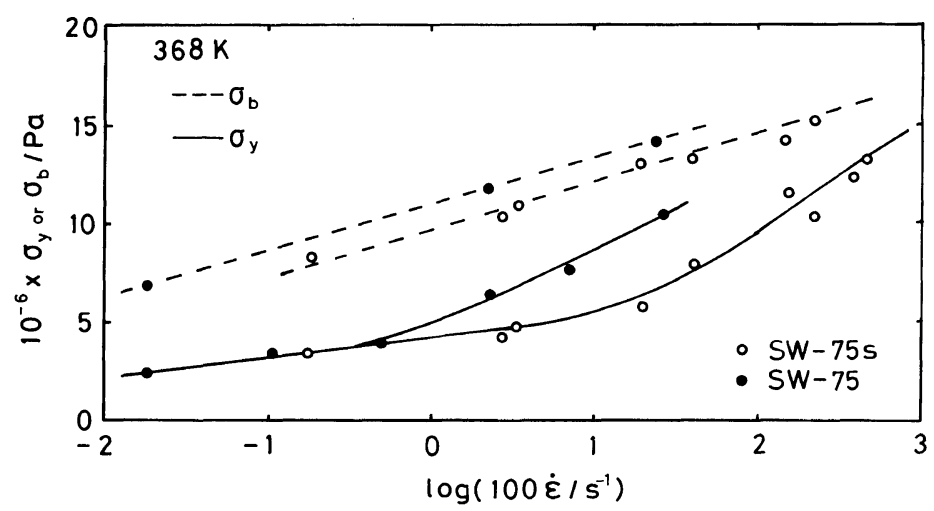

Figure 12. Plots of yield stress $\sigma_{\mathrm{y}}$ and tensile strength $\sigma_{\mathrm{b}} v s . \log \varepsilon$ for semi-IPN (SW-75s) and full-IPN (SW-75) obtained at $368 \mathrm{~K}$.

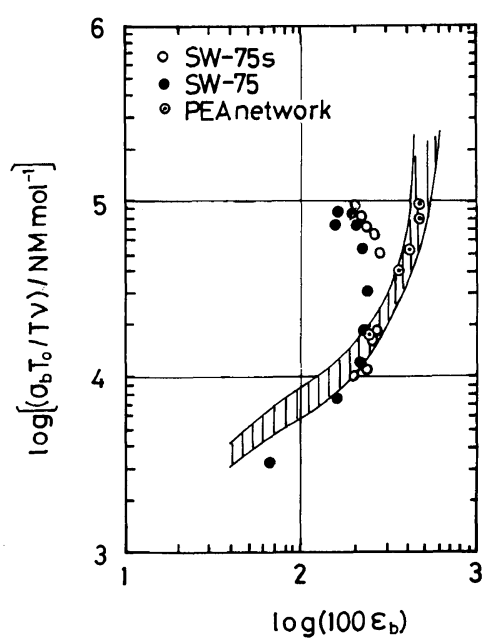

Figure 13. Reduced failure envelopes, $\log \left(\sigma_{\mathrm{b}} T_{0} / v T\right)$ vs. $\log \left(100 \varepsilon_{\mathrm{b}}\right)$, for PEA (I) network, SW-75s, and SW-75. Shaded area indicates the region where most of homopolymer-elastomer data fall. ${ }^{27}$

about a $10 \%$ conversion. During this stage PMMA microgels of various sizes are presumably formed within the (I) network. The PMMA microgels are eventually interconnected and develop into a fully interpenetrating (II) network having microheterogeneous nodular structure. However, if the reaction terminates at an early stage as was the case for CV-10 IPN, the PMMA (II) network may not have developed fully with PMMA microgels left dispersed in the PEA (I) network. The PMMA microgels might act as filler particles for the rubbery PEA (I) network. This model is supported by the facts that $\mathrm{CV}-10,27$, and 36 IPNs are all opaque, that the $E^{\prime \prime}$ and $\tan \delta$ curves for $C V-10$ possess distinct two peaks at the $T_{\mathrm{g}}$ 's of PEA and PMMA, and that the CV-IPNs have larger tensile strength than the corresponding SW-IPNs. On the other hand, in all SW-IPNs the structure seems to be more homogeneous, since in the latter all MMA monomers eventually polymerize and crosslink by the end of the reaction. The extent of the interpenetration of PEA and PMMA networks in the SWIPNs is greater than the corresponding CV-IPNs.

\section{Comparison of Semi- and Full-IPNs}

The data given above indicate that when deformation is small, the properties of the semi- and fullIPNs, SW-75s and SW-75 are almost the same. The effective network chain densities $v$ as determined from Young's moduli of PEA (I) network, PMMA homonetwork, SW-75s and SW-75 are compared in Table II. TAble II also lists the $v$ values calculated by Sperling's equation ${ }^{28}$ which assumes additivity in $v$ of the (I) and (II) networks. The difference between observed $v$ of SW-75s and SW-75 roughly corresponds to that of PMMA homonetwork. However, the large differences between the calculated and observed $v$ for both SW-75s and SW-75 suggest that in semi- and full-IPNs extensive entanglements take place between the PEA and PMMA chains. Small deformation viscoelastic behavior of both IPNs is thermorheologically simple judging from the fact that $\log a_{\mathrm{T}}$ is well described by the WLF equation. These results imply that the interpenetration of the two components is fairly good. 
Table II. Network chain densities of PEA network, PMMA homonetwork, semi- and full-IPNs both with $75 \mathrm{wt} \%$ PMMA

\begin{tabular}{|c|c|c|c|}
\hline \multirow{2}{*}{ Sample } & \multirow{2}{*}{$\begin{array}{l}\text { Young's modulus }^{\mathrm{a}} \\
E / \mathrm{MPa}(\mathrm{Temp})\end{array}$} & \multicolumn{2}{|c|}{$\begin{array}{l}\text { Effective network chain density } \\
\qquad v \times 10^{-2} / \mathrm{mol} \mathrm{m}^{-3}\end{array}$} \\
\hline & & Observed $^{\mathrm{b}}$ & Calculated $^{\mathrm{c}}$ \\
\hline PEA network & $0.19(291 \mathrm{~K})$ & 0.26 & \\
\hline PMMA homonetwork & $0.51(428 \mathrm{~K})$ & 0.48 & \\
\hline Semi-IPN(SW-75s) & $1.72(413 \mathrm{~K})$ & 1.67 & 0.16 \\
\hline Full-IPN(SW-75) & $2.02(413 \mathrm{~K})$ & 1.96 & 0.52 \\
\hline \multicolumn{4}{|c|}{$\begin{array}{l}\text { Determined from the initial slope of stress-strain curve. } \\
\text { Determined as } v=E / 3 R T \text {. } \\
\text { Calculated from the following equations }{ }^{28}: v_{\text {semi-IPN }}=v_{\mathrm{PEA}} \times V_{\mathrm{PEA}}^{1 / 3} \text { and } v_{\text {full-IPN }}=v_{\mathrm{PEA}} \times V_{\mathrm{PEA}}^{1 / 3}+v_{\mathrm{PMMA}} \times V_{\mathrm{PMMA}} \\
\text { with } V_{\mathrm{m}}(m=\mathrm{PEA} \text { or PMMA) the volume fractions. }\end{array}$} \\
\hline
\end{tabular}

Significant differences become apparent for large and high speed deformation behavior of semi and full-IPNs. In creep tests, nonlinearity becomes apparent in SW-75s sooner. On the other hand, in tensile tests, especially at high strain rates, SW-75 is stiffer and stronger. The larger network chain density and the mutual interpenetration of the two networks in the full-IPN restrict the molecular motion and prevent plastic flow from taking place. The high network chain density leads to linear behavior when the stresses or deformation is small and also imparts strength to the system.

Acknowledgment. We acknowledge with gratitude the support from the Ministry of Education, Science, and Culture under grants 147087 during 1976-1977 and 543026 during 1980-1981.

\section{REFERENCES}

1. J. R. Millar, J. Chem. Soc., 1311 (1969).

2. H. L. Frish, K. C. Frish, and D. Klempner, "Chemistry and Properties of Crosslinked Polymers," S. S. Labana, Ed., Academic Press, New York, 1976, p 205.

3. J. A. Manson and L. H. Sperling, "Polymer blends and Composites," Plenum Press, New York, 1976.

4. L. H. Sperling, J. Polym. Sci., Macromol. Rev., 12, 141 (1977).

5. L. H. Sperling, J. Polym. Sci., Polym. Symp., No. 60, 175 (1977).

6. D. A. Thomas and L. H. Sperling, "Polymer Blends," Vol. 2, D. R. Paul and S. Newman, Ed., Academic Press, New York, 1978, p 1.

7. L. H. Sperling and D. W. Friedman, J. Polym. Sci.,
$A-2,7,425$ (1969).

8. L. H. Sperling, D. W. Taylor, M. L. Kirkpatrick, H. F. George, and D. R. Bardman, J. Appl. Polym. Sci., 14, 73 (1970).

9. L. H. Sperling, H. F. George, Volker Huelck, and D. A. Thomas, J. Appl. Polym. Sci., 14, 2815 (1970).

10. Volker Huelck, D. A. Thomas, and L. H. Sperling, Macromolecules, 5, 340 (1972).

11. Volker Huelck, D. A. Thomas, and L. H. Sperling, Macromolecules, 5, 348 (1972).

12. A. A. Donatelli, L. H. Sperling, and D. A. Thomas, Macromolecules, 9, 671 (1976).

13. A. A. Donatelli, L. H. Sperling, and D. A. Thomas, Macromolecules, 9, 676 (1976).

14. L. Bohn, Rubber Chem. Technol., 41, 495 (1968).

15. L. J. Hughes and G. L. Brown, J. Appl. Polym. Sci., 5, 580 (1961).

16. P. Bauer, J. Henning, and G. Schreyer, Angew. Makromol. Chem., 11, 145 (1970).

17. H. Sumitomo and Y. Yahama, Kogyo Kagaku Zashi, 55, 179 (1952).

18. T. G Fox, Bull. Am. Phys. Soc., 1, 123 (1956).

19. G. Kraus and K. W. Rollman, "Multicomponent Polymer Systems," Vol. 99, N. J. Platzer, Ed., Adv. Chem. Ser., Amer. Chem. Soc., Washington, D. C., 1971, p 189.

20. W. E. A. Davies, J. Phys. D, Appl. Phys., 4, 1176 (1971).

21. G. Allen, M. J. Bowden, S. M. Todd, D. J. Blundell, G. M. Jeffs, and W. E. A. Davies, Polymer, 15, 28 (1974).

22. G. Allen, M. J. Bowden, D. J. Blundell, F. G. Hutchinson, G. M. Jeffs, and J. Vyvoda, Polymer, 14, 597 (1973).

23. See, for example, L. Nielsen, "Predicting the Properties of Mixtures," Marcel Dekker, Inc., New York, 1978.

24. M. Takayanagi, J. Appl. Polym. Sci., 10, 113 (1966). 
25. J. D. Ferry, "Viscoelastic Properties of Polymers," 3rd ed., John Wiley, New York, 1980.

26. T. L. Smith, J. Polym. Sci., A, 1, 3597 (1963).

27. R. F. Landel and R. F. Fedors, "Deformation and Fracture of High Polymer," H. K. Kausch, J. A.
Hassel, and R. I. Jaffee, Ed., Plenum Press, New York, 1972, p 131.

28. D. L. Siegfried, D. A. Thomas, and L. H. Sperling, Macromolecules, 12, 586 (1979). 